\title{
Industrial polysaccharides
}

\author{
Iain C M Dea \\ Leatherhead Food Research Association, Leatherhead, Surrey KT22 7RY, \\ UK
}

\begin{abstract}
Polysaccharides are widely used in the food industry as functional ingredients. Although a few act as emulsifiers, their main application is in the rheological control of the aqueous phase. Many polysaccharides perform as simple thickeners. Such materials are low cost commodities, and in general the cheapest find greatest application. Of greater interest are those polysaccharides which are able to confer novel rheological properties to the aqueous phase. In recent years a good understanding of the relationship between polysaccharide structure and rheological functional properties has been developed. As a result it is now recognised that the biotechnological talloring of polysaccharide structure can result in an optimisation of functional properties.
\end{abstract}

\section{INTRODUCTION}

Polysaccharides are widely used in the food industry as functional ingredients. Although a few polysaccharides are surface active and act as whipping agents and emulsifiers (e.g. methyl-celluloses), their main application is in the rheological control of the aqueous phase. The chemical structures of polysaccharides are of prime importance in determining their solution properties. This can be appreclated by comparing some common homoglucans. Thus cellulose ( $\beta$ 1,4-1inked D-glucan) is water insoluble and highly crystaline. Amylose ( a 1,4-linked $\mathrm{D}$-glucan) is sparingly soluble in water, crystallises less well than cellulose, and can form rigid thermoroversible gels at low concentration. In contrast, dextran ( 1,6-1inked D-glucan) is extremely water soluble and non-gel forming.

The reason that polysaccharides can exhibit large differences in solubility, and in their rheological control of the aqueous phase, is because their different primary chemical structures determine the shapes they can adopt in aqueous systems. A knowledge of polysaccharide molecular shape, and of the potential polysaccharides have for intermolecular interactions, is essential for the understanding and control of rheologiclal properties in food applications.

Many polysaccharides perform as simple thickeners. Here the polysaccharide molecules exist as fluctuating disordered chains (random coil). Their viscosity behaviour is non-specific, in that when molecular weight is normalised for, a general pattern describing the shear dependence and the concentration dependence for all polysaccharides of this type can be seen (ref. 1). The break in the plot in Fig. 1 is at the transition from dilute solution behaviour (low degree of coil overlap) to concentrated solution behaviour (total interpenetration of random coll molecules). Polysaccharides which are exploited as simple thickeners in the food industry include sodium alginate, carboxymethyl-cellulose, the plant seed galactomannans, and $\lambda$-carrageenan. Such materials are low cost commodities. They differ from each other quantitatively rather than qualitatively, and in general the cheapest find greatest application.

Of greater interest are those polysaccharides whlch confer novel rheological properties, such as rigid gel formation or weak gel properties, to the aqueous phase. While thickened polysaccharide solutions are related to the properties of disordered polymer chains interacting by virtue of entanglements, the origin of rigid and weak gel properties is specific molecular association of polymer chains. In recent years a good understanding of the relationship between polysaccharide structure and these novel rheologiclal properties has been developed. As a result there is now an opportunity to tallor polysaccharide structure to optimise these structure/function relationships. 


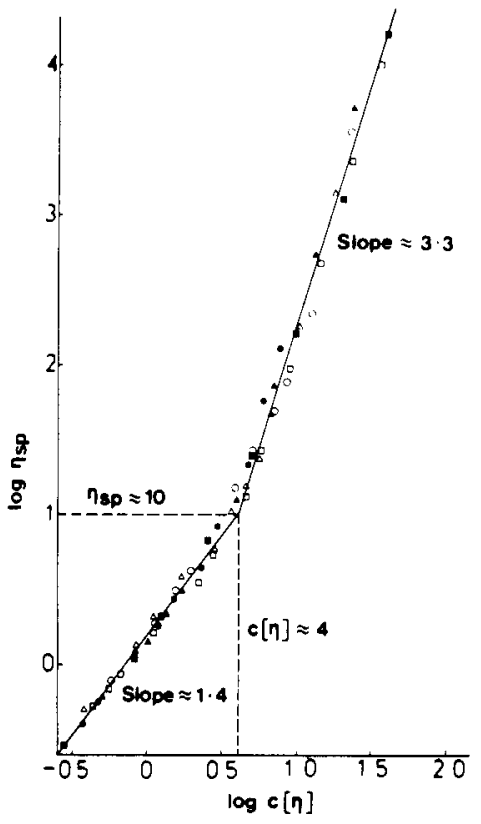

Fig. 1. Generalised concentration dependence of specific viscosity for a range of polysaccharides

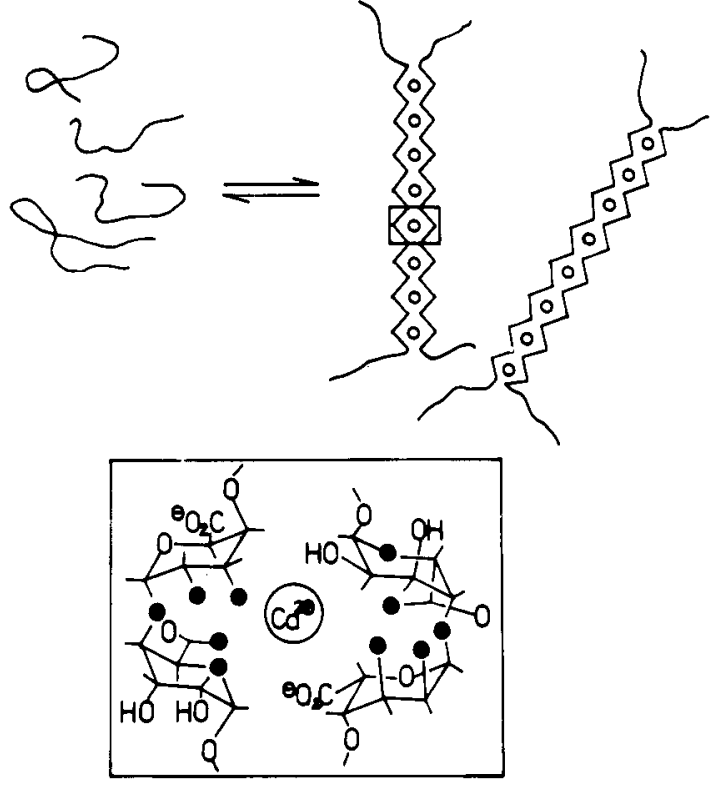

Fig. 2. Representation of the association of polyguluronate sequences by chelation of calcium

\section{MOLECULAR ORIGIN OF POLYSACCHARIDE GEL FORMATION}

\section{Aggregation of ordered ribbon conformations}

Alglnates are a group of acidic polysaccharides from brown seaweed, which gel by co-operative binding of divalent cations in particular calcium. They are linear polymers of 1,4-p-D-mannuronic acld and 1,4-a-L-guluronic acid, which contain homopolymeric sequences of D-annuronate and I-guluronate together with reglons in which the two sugars alternate (I). The relative proportion of these three different "blocks" varles with biological source. A combination of cation binding and circular dichrolsm measurements has led to the conclusion that the mechanism of cross-1inking in alginate gels involves co-operative binding of crlclun lons between aligned polyguluronate ribbons (ref. 2). This is the well known "egg box" model (Fig. 2). It is reasonable to assume that the ordered conformation of the polyguluronate sequences in these function zones is similar to the buckled two-fold structures determined for polyguluronate salts in the solid state (ref. 3 ). Competitive Inhibition studies suggest a gelation mechanism based on dimerisation of these ordered polyguluronate conformations (ref. 2 ).

Low-wethoxy pectin is the partial methyl ester of 1,4-11nked poly-a-D-galacturonic acid. The structure is, however, complicated by interuptions in the chain by single 1,2- $a$ -L-rhamose residues (II). Low methoxy pectins are prepared from natural high methyl pectins by a sonification process which lowers the level of methyl esterification from $70 \%$

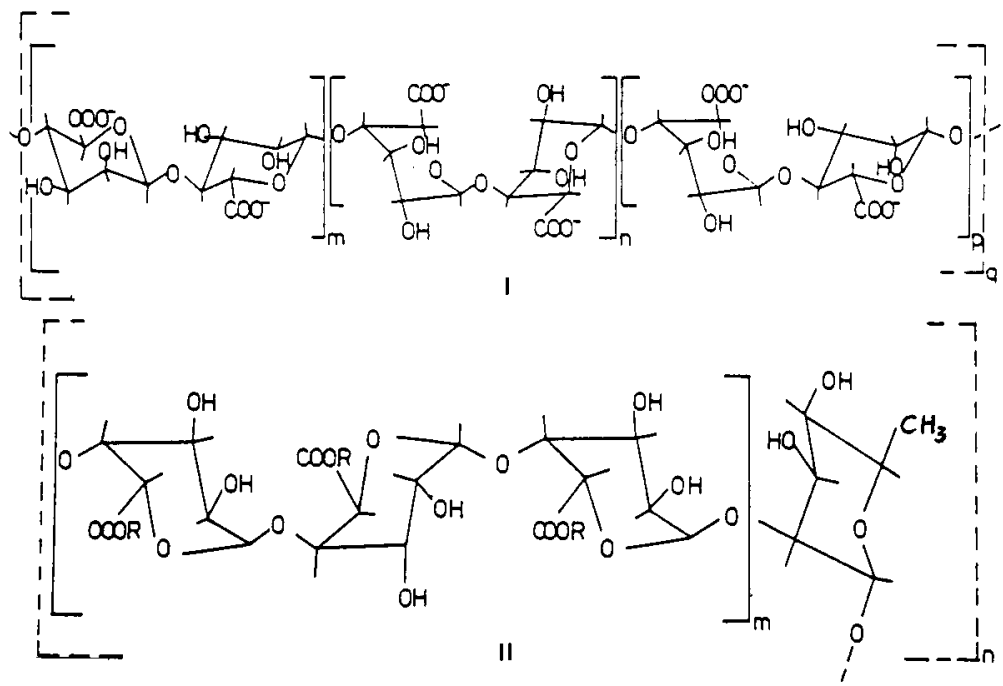


to ca. 30\%. The calcium binding behaviour of low methoxy pectin is very simflar to that of the polyguluronic "blocks" of alginate, and low methory pectin froms firm calciun gels in the same way as alginate. This 18 not altogether surprising since the polygalacturonate structure is almost the exact nirror image of polyguluronate except at carbon 3 , and similar binding behaviour mlght therefore be expected (III). The mechanism of cross-1inking in low methoxy pectin gels can therefore also be cons1dered in terms of the "egg box" model shown in Fig. 2 .

Natural high-methoxy pectins show less affinity for calcium. They do, however, gel under conditions of low $\mathrm{pH}$ and low water activity. High methoxy pectin gelation requires sucrose concentrations in excess of $65 \%$. The resultant reduction in water activity makes the system a poorer solvent. Solvent-polymer interactions are reduced and polymer chain-chain associations are favoured. However, this increase in polymer interaction is not sufficient to result in gel formation. This is because there are charge-charge repulsions between the negatively charged pectin molecules, which prevent complete network formation. This is overcome by a reduction in $\mathrm{pH}$ to less than 4.0 , which suppresses the lonisation of the carboxyl functions. Although less is known about the mechanism of cross linking in these low water activity pectin gels, the absence of any detectable competitive inhibition of network formation by addition of short chain "blocks" suggests that the cross-1inking is through aggregates of ordered ribbon conformations of variable extent, rather than junction zones of fixed stoichiometry.

A number of neutral polysaccharldes also form gels under low water activity conditions. In this case low $\mathrm{pH}$ values are not a prerequisite. Notable examples are the amyloid from Tamarindus indica (a substituted cellulose) and certain plant seed galactomannans (polysaccarides based on a 1,4- $\beta-D-$ mannan main chain solubilised by substitution with $1,6-a$ -D-galactose stubs). Firm gelation can be induced by the use of high concentrations of sucrose or ethylene glycol. X-ray diffraction analysis of galactomannans indicate that in the condensed state the conformation of the molecule is very similar to that of mannan (ref. 4). Similarly, amyloids exhibit closely similar $x$-ray diffraction patterns to cellulose. The mechanism of gelation of these solubilised neutral celluloses and mannans is likely to involve chain-chain associates by aggregation of regular ordered cellulose or mannan backbones. For galactomannans there is evidence for a correlation between galactomannan structure (1.e. degree of galactose substitution and amount and length of unsubstituted mannose regions) and the ability to form gels at low water activity (refs. 5,6). Thus the gel junction zones probably comprise aggregates of regular unsubstituted regions of mannan backbone, while the more heavily substituted regions of the molecules serve to solubilise the network (Fig. 3).

The primary structure of xanthan consists of a cellulose backbone, substituted on alternate residues with trisaccharide side chains so that the repeating unit is a pentasaccharide (IV). It is the presence of this regular substitution that confers solubility to xanthan.
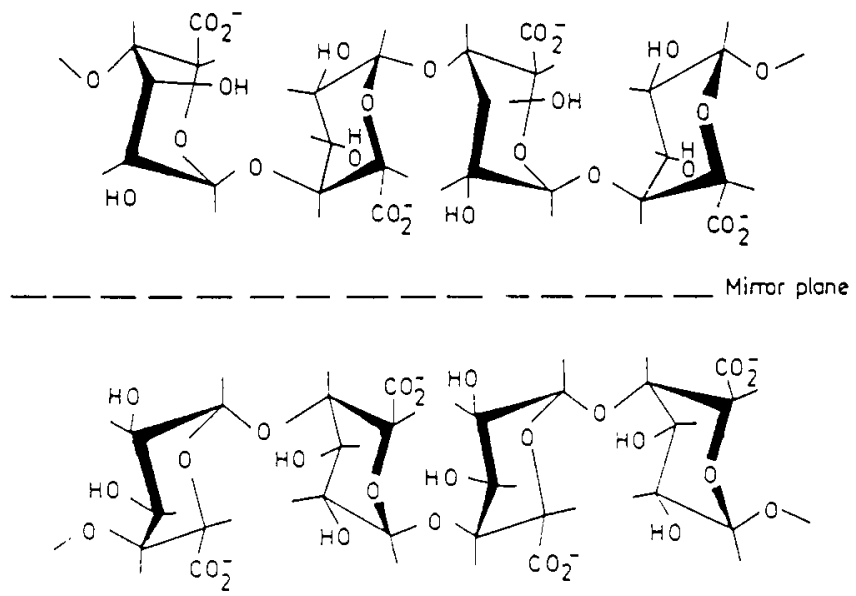

III

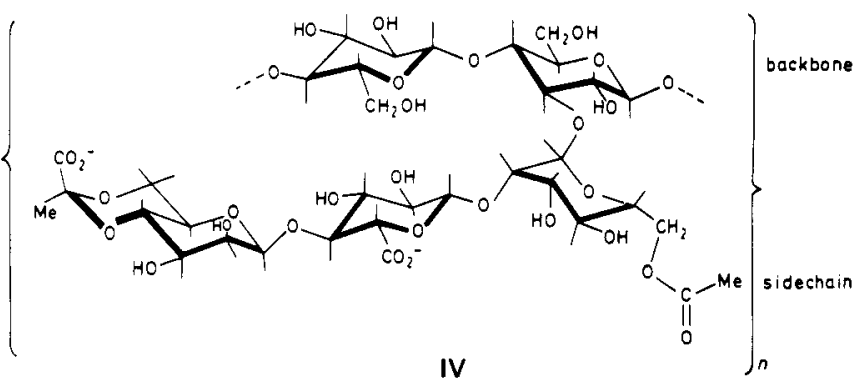

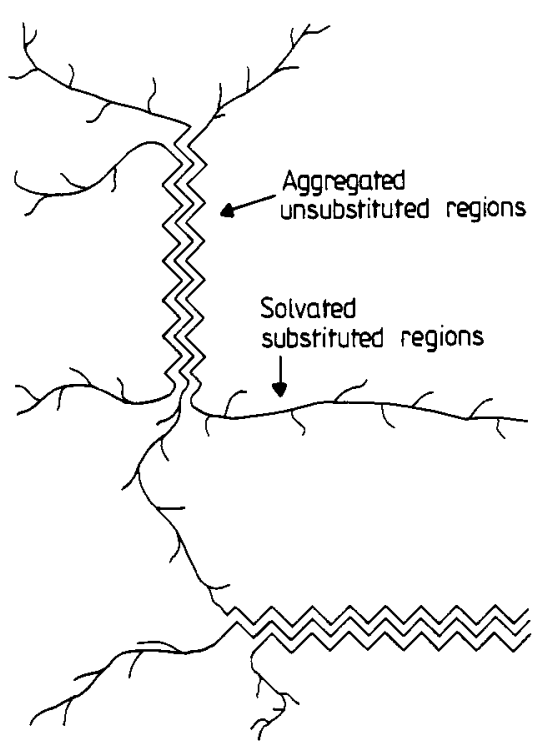

Fig. 3. Proposed model of galactomannan ge/ structure 


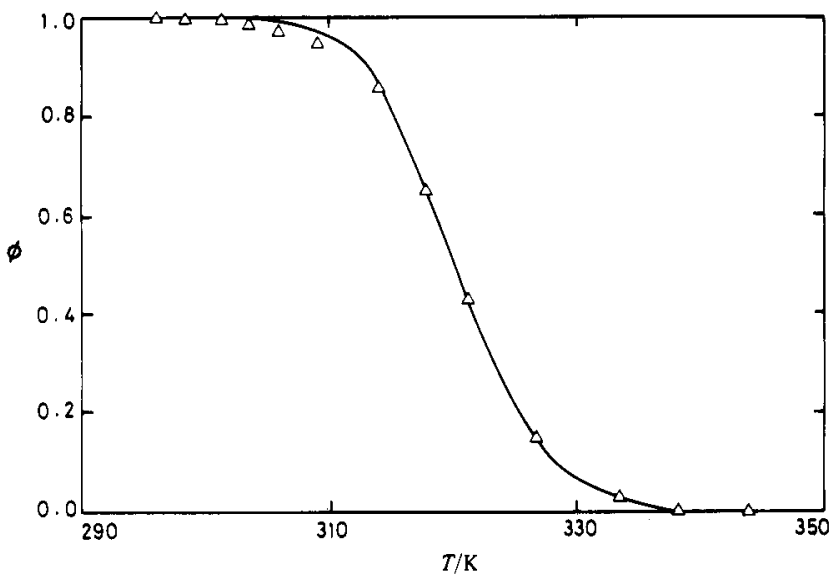

Fig. 4.

Generalised representation of the temperature dependence of the order-disorder transition for xanthan, where $\phi$ represents the fraction of the residues in the ordered conformation

In contrast to the amylolds and galactomannans, xanthan exhibits weak gel properties in aqueous systems. Evidence from N.m.r., optical rotation, circular dichroism and D.S.C. indicates that santhan undergoes an order-disorder transition in aqueous solution with change in temperature, which exactly parallels the temperature dependent formation and melting of the weak gel properties (ref. 7). This temperature dependent order-disorder transition shows no thermal hysteresis, and may be represented by plotting the fraction of the residues in the ordered conformation against temperature (Fig. 4). X-ray diffraction studies (ref. 8) and stopped-flow polarimetry (ref. 7) argue strongly that the ordered conformation of xanthan is single stranded, and it is generally accepted that the weak gel properties of xanthan are built up by the alignment, aggregation and tenuous association of these rigid single stranded molecules in solution to give a three-dimensional structure.

\section{Multiple helices in gel network formation}

1- and $k$-Carrageenan are polysaccharides from red seaweeds which form rigid thermoreversible gels. They are alternating copolymers comprising 3-linked- $\beta-D-g a l a c t o s e$ and $4-1$ inked 3,6-anhydro-a-D-galactose. i-Carrageenan is the more highiy sulphated, bearing two sulphate groups per disaccharide repeating unit, compared with $k$-carrageenan which contains one sulphate groups per repeating unit $(V)$. A range of spectroscoplc techniques has indicated that gel melting and setting is parallelled by a temperature dependent order-disorder transition. X-ray fibre diffraction has indicated that both l-carrageenan (ref. 8) and $k$-carrageenan (ref. 9) exist as 3-fold right-handed double helices in the solid state, while optical rotation and stopped-flow polarimetry (ref. 10) argue strongly for this ordered conformation being involved in gel formation. The aggregation of these double helical ordered conformations in aqueous systems depends on factors such as sulphate content of the carrageenan, lonic strength and cation type present. Gel formation only occurs under conditions where aggregation of double helices can occur (Fig. 5). Because $k$-carrageenan carrles less negative charge than t-carrageenan, it shows a greater degree of double helix aggregation, and its gels are therefore stronger and more opaque.

Agarose has a similarly alternating disaccharide structure to the carrageenans, comprising 3-1inked- $\beta-D$-galactose and 4-1inked 3,6-anhydro-a-I-galactose (VI). X-ray fibre diffraction (ref. 11) has indicated that agarose exists as 3-fold left-handed double helices in the solid state, in contrast to right handed double helices for the carrageenans. Agarose, a

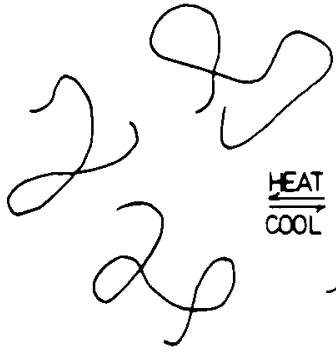

Random coils

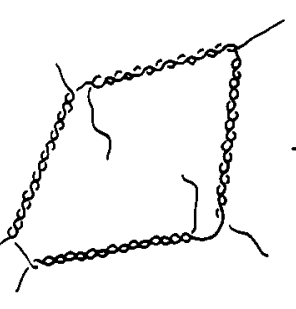

Ordered "domains"

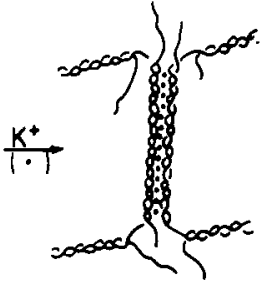

Aggregated "domains"

Fig. 5. Domain model of carrageenan gelation

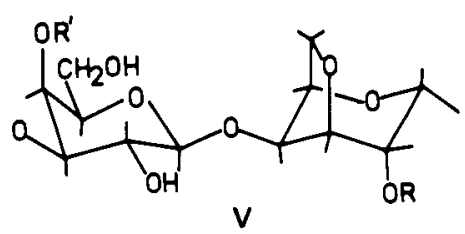

$\mathrm{R}=\mathrm{H}, \mathrm{R}^{\prime}=\mathrm{SO}_{3} \mathrm{H}$ for $\mathrm{k}$-carrageenan $R=R^{\prime}=\mathrm{SO}_{3} \mathrm{H}$ for $i$-carrageenan

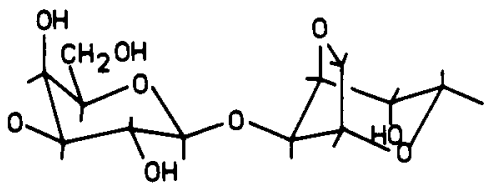




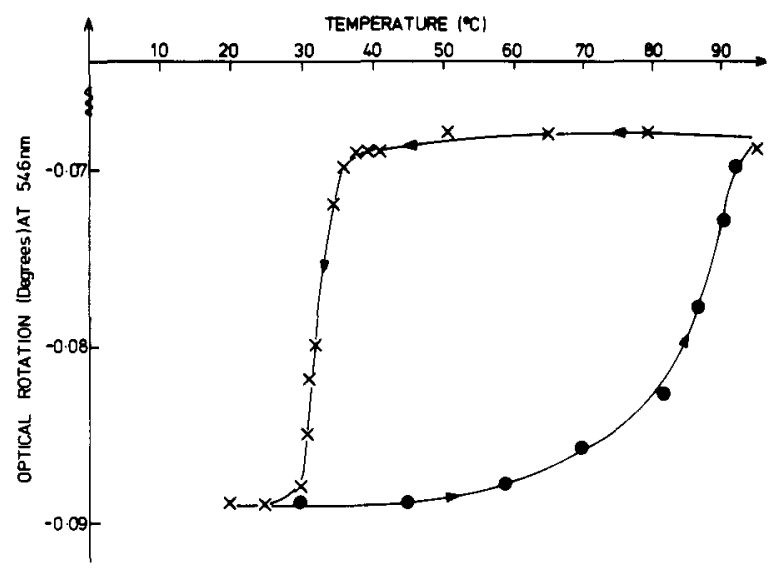

Fig. 6. Variation of optical rotation with temperature for $0.2 \%$ agarose

neutral polysaccharide, forms very rigid thermoreversible gels at low concentrations. An optical rotation transition is observed for the setting and melting of agarose, indicating a temperature dependent order-disorder transition (Fig. 6). The sign and magnitude of this optical rotation transition is consistant with the involvement of aggregated double helices in the formation of the gel network.

A number of natural agar variants exist which involve substitution with 0-methyl, 0-sulphate and pyruvic acid ketals. The extent of hysteresis for the optical rotation transition varies quite markedly for different substituted agars. The important factor here appears to be the degree of substitution by negatively charged groups. Thus the extent of hysteresis decreases on going from agarose through the pyruvic acid substituted agar from Gracilaria compressa, to the highly sulphated agar from Gliopeltis cervicornis. It is reasonable to interpret these differences as arising from changes in charge density of the polysaccharide chain altering the degree of aggregation of the double helices in the gel network. As the degree of aggregation in the gel network increases, so the gels become more turbid, more brittle, and show increased hysteresis behaviour on melting and setting.

Since the demonstration that double helix formation is involved in the gelation of the agars and carrageenans, it has become clear that this mechanism of gel formation is quite widespread. For example the gelation mechanism for amylose (the linear a 1,4-D-glucan component of starch) involves aggregation of the double helical ordered conformation, while that of curdlan ( $1,3-\beta-D$-glucan) involves aggregation of the triple helical ordered conformation. It is also possible that gellan gum, one of the more recently introduced industrial polysaccharides, may have a similar gelling mechanism. Gellan gum is the entracellular polysaccharide from Pseudomonas elodea, which has been shown to have a regular tetra-saccharide repeating unit containing one uronic acid residue (ref. 12). At low concentration, it forms firm, brittle, aqueous gels whose strength and thermal stability are influenced by the type and concentration of cations present. X-ray fibre diffraction has recently indicated that gellan gum exists as a left handed three-fold double helix in the solid state (ref. 13), and it is possible that this ordered conformation is the basis of the gelation of gellan gum.

\section{Mixed polysaccharide interactions}

There are numerous examples where the interaction between two polysaccharides can be industrially exploited to confer weak or rigid gel properties to the aqueous phase. Here the resultant rheology is a consequence of specific interaction between the polysaccharide components, rather than a simple additive effect. Soluble 1,4-linked $\beta-D$-glycans, in particular the galactomannans, play a central role in these interactions. Under conditions where they do not exhibit rigid or weak gel propertles, they can enhance the nove 1 rheological properties of certain other polysaccharides when added at low levels (ref. 14).

Key examples of this gelation mechanism are the interactions of agars, carrageenans and xanthan with the galactomannans. Thus, addition of low levels of locust bean gum, the galactomannan from Ceratonia siliqua in which approximjately $30 \%$ of the mannose residues in the mannan chain are substituted by a-D-galactose residues ( $23 \%$ galactose, $77 \%$ mannose), causes non-gelling concentrations of agarose $(0.05 \%)$ and $k$-carrageenan (1\%) to form firm rigid gels. Furthermore, xanthan alone, at all accessable concentrations, confers weak gel properties to aqueous systems, but on admixture with locust bean gum firm rigid gels are produced.

The extent of these gelling interactions is controlled and modified by chemical structure variations. Thus in the case of the agars, the non-substituted agarose exhibits the strongest gelling interaction with galactomannan. The higher the degree of natural substitution by 0 -methyl, 0-sulphate and pyruvic acid ketal groups in agar varients the less the gelling interaction with galactomannans. The gelling interaction between agars and 
galactomannan can be monitored by measuring the temperature dependent changes in optical rotation, and Fig. 7 illustrates this effect of increasing substitution in the agar molecule (ref. 15). A simllar phenomenon is observed in the carrageenan serles with the extent of 0-sulphation. Furcellaran, which contalns half the level of 0 -sulphation of $k$-carrageenan gels better with locust bean gum, while l-carrageenan, which contains twice the level of 0-sulphation exhibits no gelation interaction whatsoever with galactomannans (ref. 15). The mixed gelling interaction between xanthan and galactomannans is similarly affected by changes in the structure of xanthan. Thus deacetylation of xanthan significantly increases the gelling interaction with galactomannans (ref. 16).

Structural variations in the galactomannan molecule also affect these important gelling interactions. In general, the most effective galactomannans in these co-gelling systems are those in which the mannan backbone is least substituted by D-galactose. The distribution of D-galactose substituents along the mannan backbone also plays an important role. It is now known that in some galactomannans (e.g. Caesalpinia pulcherima galactomannan) the galactose distribution along the main chain is closely similar to statistically random, while for others (e.g. locust bean gum) there is a non-regular non-statistically random distribution of galactose, resulting in a higher proportion of unsubstituted blocks in the main chain of intermediate length (refs. 5,17,18). Galactomannans with a larger proportion of longer reglons of unsubstituted blocks or sides along the mannan backbone interact best with agars, carrageenans and xanthan, and this is consistent with the proposal that the molecular origin of these gelling interactions is the ordered binding of unsubstituted extended ribbon conformation of the mannan backbone with the double helical regions of agars and carrageenans or the single-stranded ordered conformation of xanthan (Fig. 8).

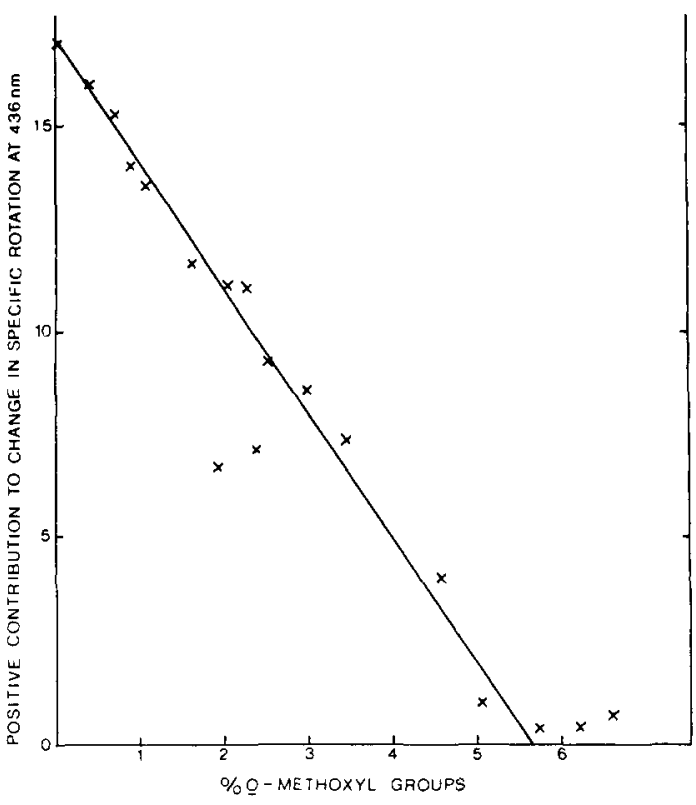

Fig. 7. Comparison of the positive contribution to change in optical rotation due to locust bean gum on cooling mixtures of agarose (0.1\%) and locust bean gum (0.3\%) with variation in OMe content of the agarose

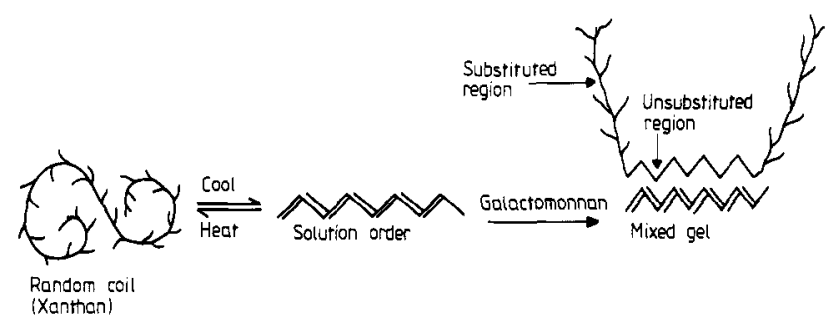

Fig. 8. Model for the interaction between xanthan and galactomannan

TABLE 1. Propertles of modified alginates

\begin{tabular}{|l|c|c|}
\hline Sample & $\begin{array}{c}\text { Guluronic Acld } \\
\text { Content (\%) }\end{array}$ & $\begin{array}{c}\text { Gel Strength* } \\
\left(\mathrm{Ncm}^{-2}\right)\end{array}$ \\
\hline Native M.pyrifera & 39 & 4.9 \\
Modified M.pyrifera & 62 & 6.8 \\
Native L.digitata & 41 & 4.0 \\
Modified L.digitata & 62 & 9.2 \\
\hline
\end{tabular}

* $2 \%$ calcium alginate gels

\section{TAILORING OF POLYSACCHARIDE STRUCTURE}

Because of this well developed understanding of the relationship between polysaccharide structure and novel rheological properties, we are now in the position to attempt to tailor polysaccharide structure in order to optimise these structure/function relationships. Targets which might be set include the modification of structure to optimise properties, the structure modification of a cheap material to match the structure and properties of a more expensive alternative, and the development of new structures which might be expected to exhibit desirable propertles. The blotechnological techniques of enzyme technology and fermentation are beginning to be exploited in this area.

\section{Enzymic upgrading of polysaccharides}

As mentloned earller calcium gelation of alginates involves the alignment and ordered binding of guluronic acid blocks within the alginate molecules. Correct selection of seaweed species and harvesting of the correct parts of the weeds can ensure high contents of guluronic acld blocks, and therefore optimal gel properties. Biotechnology of fers the opportunity of upgrading any high molecular weight native alginate to a good gel former. 
This approach involves the use of mannuronan-C-5 epimerase which epimerases D-mannuronic acid to L-guluronic acid at the polymer level (ref. 19). The enzyme preparation has to be rigorously free of the chaln splitting alginate lyase, and the conversion is carried out at $\mathrm{pH} 7.0$ in the presence of calcium 1ons. It has been demonstrated that significant increases in guluronic acid content of alginate can be achieved by this approach, and that this modification is accompanied by major increases in gel strength (Table 1). This upgrading of alginate functionality is extremely promising, and could be the basis of the supply of high grade alginate in the future.

Galactomannans play a key role in mixed gelation with other polysaccharides. As indicated earlier, those galactomannans with least galactose substituents are most effective in this situation. There are two galactomannans of commerclal significance. Guar gum which has a high galactose content, has limited functionality and is cheap, and locust bean gum which has a low galactose content, is highly functional and is expensive. It has been demonstrated that guar gum can be upgraded by enzymatic galactose depletion using plant a-galactosidase, so long as the preparation is virtually free of endo- $\beta$-mannanase (ref. 20). The galactose content can be reduced to values lower than that of native locust bean gum. Two factors affect the functionality of these galactose-depleted guar gums; they are higher in molecular weight than locust bean gum but the distribution of galactose units along the chain is almost random. In the event modified guar gum with a galactose content equal to locust bean gum is slightly less functional in mixed polysaccharide interaction than locust bean gum. There is a good chance that this development could be commercial exploited in the future since it has been demonstrated that the modifications can be carried out at very low moisture contents, so decreasing the energy costs (ref. 21).
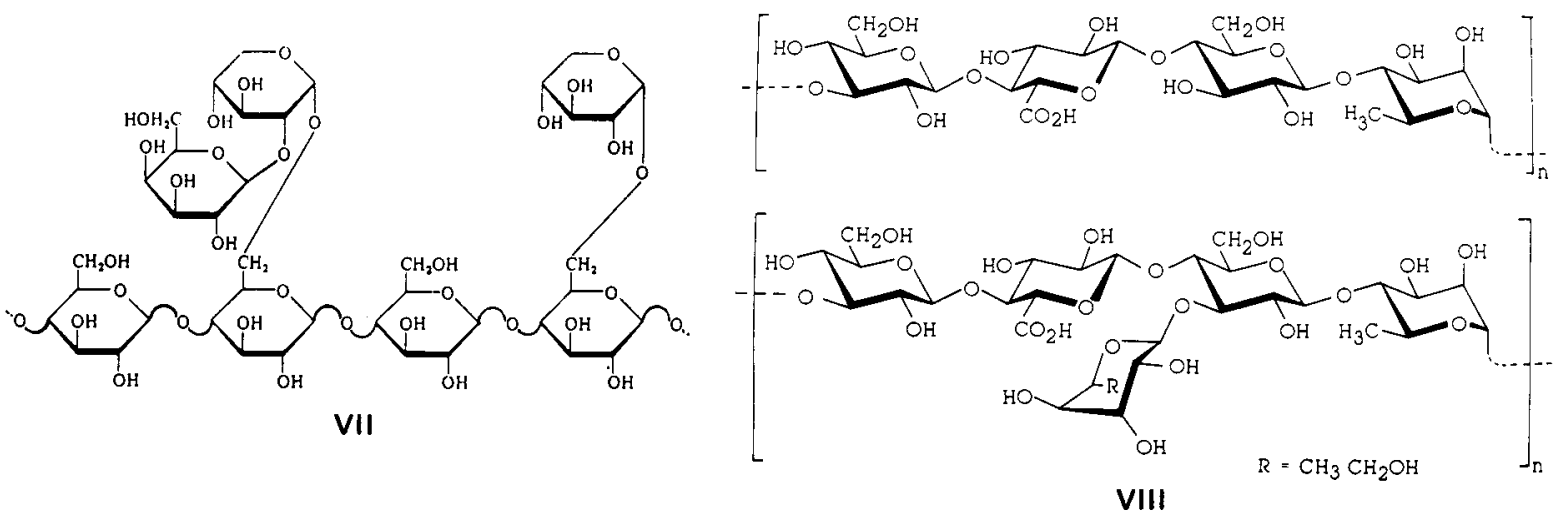

Tamarind gum, the heavily substituted natural cellulosic (VII) exhibits a very low level of mixed gelling interaction with other polysaccharides. Although no natural varients of Tamarind gum with lower levels of substitution are known, it is reasonable to propose, by analogy with galactomannans, that controlled removal of the galactose and xylose would lead to a new molecule with improved mixed gelling functionality. Initial work using an extremely pure preparation of a plant $\beta-D$-galactosidase led to the observation that depletion of half the galactose led to self gel formation (ref. 22). It would therefore appear that galactose substitution in Tamarind gum solubilises the xylogiucan structure in the same way as galactose substitution in galactomannans solubilises the mannan backbone. Indeed, galactose depleted galactomannans precipate/ge1 at the same d.s. of galactose as galactose-depleted Tamarind gum. It now needs to be determined whether these enzymiclally modified materials exhibit useful mixed gelling properties.

\section{Control of polysaccharide structure by fermentation}

Just as polysaccharide structures can be tailored enzymatically, so fermentation can be explotited. An example worthy of note is the gellan family of polysaccharides (VIII). Gellan gum and welan gum differ only in the regular substitution, by either a-I-rhamnose or $a-I$-mannose, on 0-3 of every D-glucose residue after the uronic acid in the case of welan gum. This structural modification results in a number of major differences between the two polysaccharides. Viscosity and dye binding experiments indicate that in dilute solution gellan gum exhibits typical polyelectrolyte behaviour while welan gum behaves like a neutral polymer (ref. 23). At higher concentrations gellan gum forms firm gels while welan gum only exhibit weak gel properties. X-ray flbre diffraction studies indicate that welan gum adopts an ordered conformation totally different from the double helical structure of gelian gum (refs. 13,24). In this case it is therefore clear that the side chain sugar alters the ordered conformation adopted by the main chain, and as a result modifles the functional properties of the polysaccharide. It is possible that this occurs by the welan gum molecule adopting a rigid rod conformation in which the charge of the uronic acid is shielded by hydrogen bonding with the side chain (Fig. 9) (ref. 25). It is therefore clear that the correct selection of micro-organism can tallor structure and therefore functional properties. 


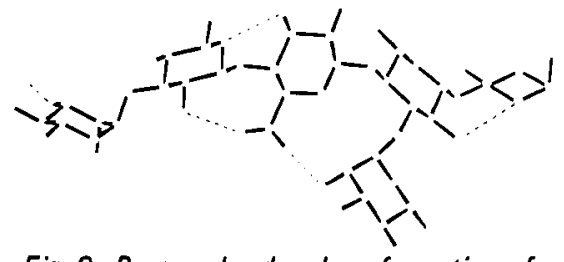

Fig. 9. Proposed ordered conformation of welan gum with $\mathrm{H}$-bonding scheme involving side chain and uronic acid in main chain

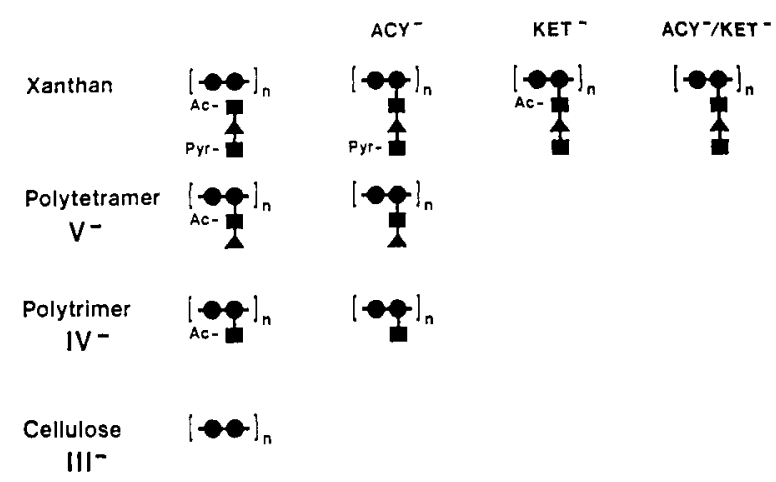

Fig. 10. Range of xanthan structural varients

An area where this approach could be exploited in the future is xanthan. It is already known that the presence or absence of 0-acetylation has a major effect on the functional properties of xanthan. What is not known, however, is the details of how the regular substition of the 0-acetylated and pyruvylated trisaccharide side chain controls the functional properties of xanthan (IV). A range of xanthomonas varients have now been developed which allow the preparation of a range of xanthan varients from an unsubstituted polytrimer up to the native substituted polypentamer (Fig. 10). This exciting development could lead to the identification of an optimised xanthan, and is a powerful illustration of how modern fermention science could be used to tailor the industrial polysaccharides of the future.

\section{REFERENCES}

1. E.R. Morris, A.N. Cutler, S.B. Ross-Murphy and D.A. Rees, Carbohydr. Polymers, 1,5-21 (1981).

2. E.R. Morris, M.J. Gidley, E.J. Murray, D.A. Powell and D.A. Rees, Int. J. Bio1. Macromol., 2, 327-330 (1980).

3. E.D.T. Atkins, I.A. Niedusynski, W. Mackie, K.D. Parker and E.E. Smolko, Biopolymers, 12, 1879-1887 (1973).

4. Y.Y. Chien and W.T. Winter, Macromolecules, 18, 1357-1359 (1984).

5. I.C.M. Dea, E.R. Morris, D.A. Rees, E.J. Welsh, H.A. Barnes and J. Price, Carbohydr. Res., 57, 249-272 (1977).

6. I.C.M. Dea, A.H. Clark and B.V. McCleary, Carbohydr. Res., 147, 275-294 (1986).

7. I.T. Norton, D.M. Gooda11, S.A. Frangou, E.R. Morris and D.A. Rees, J.Mo1.B101., 175, 371-394 (1984).

8. S. Arnott, W.E. Scott, D.A. Rees and C.G.A. McNab, J.Mo1.Bio1., 90, 253-267 (1974).

9. R.P. M1llane, R. Chandrasekaran, S. Arnott and I.C.M. Dea, Carbohydr.Res., in press:

10. I.T. Norton, D.M. Gooda11, E.R. Morris and D.A. Rees, J.Chem.Soc.Chem.Commun., 988-990 (1979).

11. S. Arnott, A. Fulmer, W.E. Scott, I.C.M. Dea, R. Moorhouse and D.A. Rees, J.MoI.B101, $90,269-284(1974)$.

12. P.E. Jansson, B. Lindberg and P.A. Sandford, Carbohydr.Res., 124, 135-139 (1983).

13. R. Chandrasekaran, R.P. Millane, S. Arnott and E.D.T. Atkins, Carbohydr.Res., 175, 1-15 (1988).

14. I.C.M. Dea, in "Recent Developments in Industrial Polysaccharides - The Impact of Biotechnology and Advanced Methodologies", ed. V. Crescenz1, I.C.M. Dea and S.S. Stivala, Gordon and Breach, New York, pp367-385 (1987).

15. I.C.M. Dea and D.A. Rees, Carbohydrate Polymers, 7, 183-224 (1987).

16. M. Tako and S. Nakamura, Carbohydr.Res., 138, 207-213 (1985).

17. B.V. McCleary, A.H. Clark, I.C.M. Dea and D.A. Rees, Carbohyd.Res., 139, 237-260 (1985).

18. I.C.M. Dea, A.H. Clark and B.V. McCleary, Food Hydrocollo1ds, I, 129-140 (1986).

19. G. Skjak-Braek, 0 . Smidsrod and B. Larsen, Int.J.Blol.Macromo1., 8, 330-336 (1986).

20. B.V. McCleary, I.C.M. Dea, J. Windust and D. Cooke, Carbohydrate Polymers 4, 253-270 (1984).

21. European Patent EP0121960.

22. J.S.G. Reld. M. Edwards and I.C.M. Dea, Gums and Stabilisers for the Food Industry 4 (Proc.), 391-398 (1988).

23. V. Crescenz1, M. Dentini and I.C.M. Dea, Carbohydr.Res., 160, 283-302 (1987).

24. P.T. Attwool, E.D.T. Atkins, M.J. Miles and V.J. Morris, Carbohydr.Res., 148, C1-C4 (1986).

25. I.C.M. Dea, in "Industrial Polysaccharides: Genetic Engineering, Structure/Property Relations and Applications", ed. M. Yalpani, Elsevier Science Publishers, Amsterdam, pp207-216 (1987).

26. M.R. Betlach, M.A. Capage, D.H. Doherty, R.A. Hassler, N.M. Henderson, R.W. . Vanderslice, J.D.Marrelli and M.B. Ward, in "Industrial Polysaccharides: Genetic Engineering, Structure/Property Relations and Applications", ed. M. Yalpani, Elsevier Science Publishers, Amsterdam, pp 35-50 (1987). 